prognostic features our series may have been, a good outcome for 3 out of 21 is very poor.

Crisp, A. H., Norton, K., Gowers, S., et al (1991) A controlled study of the effect of therapies aimed at adolescent and family psychopathology in anorexia nervosa. British Journal of Psychiatry, 159, 325-333.

Eisler, I., Dare, C., Russell, G. F. M., et al (1997) Family and individual therapy in anorexia nervosa. A 5 year follow-up. Archives of General Psychiatry, 54 1025-1030.

Gowers, S. G. \& North, C. (1999) Difficulties in family functioning and adolescent anorexia nervosa. British Journal of Psychiatry, 174, 63-66.

S. G. Gowers Section of Adolescent Psychiatry, The University of Liverpool, Pine Lodge Academic Unit, 79 Liverpool Road, Chester CHI IAW

\section{Possible causes of catatonia in autistic spectrum disorders}

I read with interest the paper by Wing $\&$ Shah (2000) on catatonia in autistic spectrum disorders. The authors quite correctly make the point that catatonia, although a useful clinical concept, is a description of a number of behaviours. However, they have not attempted to investigate the aetiology of catatonia in their sample of $40 \mathrm{pa}-$ tients. Three other possible causes for their observations spring to mind.

First, the onset of catatonic symptoms in adolescence or early adulthood, in this largely male sample, could be related to the development of schizophrenia, although it may be difficult to diagnose. It has presumably been excluded as no patients had first-rank symptoms according to the accounts of relatives or carers, although in Table 3 (p.359), the heading "bizarre/ psychotic" catatonic manifestations were found in $40 \%$ of their patients. The fact that 'Others had occasional visual hallucinations or paranoid ideas' suggests that they may qualify for an additional diagnosis of schizophrenia according to the ICD-10 (World Health Organization, 1992). The authors have not specifically stated whether the patients had been assessed for a diagnosis of schizophrenia.

Although the patients may be difficult to interview on account of communication disorders or cognitive problems, nearly half did not have impaired language and the number of mute patients is not stated. Furthermore, $70 \%$ of the patients had a level of cognitive ability within the range from mild learning disability to average intellectual ability, not incompatible with a diagnosis of schizophrenia.

Second, the possible explanation for catatonic symptoms is the development of an affective disorder. In 13 of the 30 patients, precipitating factors included bereavement, pressure at school, lack of structure after leaving school and lack of occupation, which are more commonly associated with a depressive illness. Central to the diagnosis of catatonia are increased slowness, difficulty in initiating and completing actions and lack of motivation, among others, possibly symptoms of depression.

Third, and most importantly, catatonic symptoms may be difficult to distinguish from the extrapyramidal side-effects of antipsychotic drugs (American Psychiatric Association, 1992). In Wing \& Shah's description of the criteria for catatonia, a secondary feature listed was "Parkinsonian features: tremor, eye-rolling, dystonia, odd stiff posture, freezing in postures, etc.". Although the patients are fairly young, they are also a tertiary referral group and it is likely that they would have received other, previous treatments. Recent estimates of prescriptions of psychotropic medication to adolescents and adults with developmental disabilities vary from 12 to $40 \%$ (Connor \& Posever, 1998). There was no record of previous treatment and, more specifically, a history of current or prior exposure to antipsychotics is omitted.

It is helpful to know that catatonia can complicate autistic spectrum disorders and that individuals who present with catatonia may have an undiagnosed autistic spectrum disorder. However, although recognition is necessary to institute appropriate management, this paper offers only limited help in this direction. There would have been a greater clinical impact if it had addressed the possible causes of catatonia or the other associated psychopathology. The study also raises the question of whether catatonia represents the expression of other, more common mental disorders in those with limited communication skills.

American Psychiatric Association (1992) Differential diagnosis of tardive dyskinesia. In Tardive Dyskinesia: A Task Force Report of the American Psychiatric Association, pp. 9-34. Washington, DC: APA.

Connor, D. F. \& Posever, T. A. (1998) A brief review of atypical antipsychotics in individuals with developmental disability. Mental Health Aspects of Developmental Disabilities, I, 93-102.
Wing, L. \& Shah, A. (2000) Catatonia in autistic spectrum disorders. British Journal of Psychiatry, $\mathbf{1 7 6}$ 357-362.

World Health Organization (1992) The ICD-10 Classification of Mental and Behavioural Disorders. Geneva: WHO

R. Chaplin South West London \& St George's Mental Health Trust, 6 I Glenburnie Road, London SWI7 7DJ

Authors' reply: Dr Chaplin notes that neither the possible causes nor the treatment of catatonia were discussed in our paper. As the Journal requires papers to be 3000-5000 words long, we decided to focus on the clinical picture of catatonia in autism and its prevalence. We have written and intend to publish a second paper dealing with causes and treatment and are grateful to Dr Chaplin for providing us with the opportunity to write a few more words on these subjects.

The individuals in the study had all been seen by one or more clinicians before the tertiary referral to Elliot House. During the course of the multiple assessments, possible underlying causes, including schizophrenia, depression, obsessivecompulsive disorder and identifiable brain pathology such as parkinsonism, would have been considered. These conditions, together with autistic spectrum disorders and catatonia, are defined and diagnosed only on history and clinical picture and there is overlap of clinical features among them all. In the individuals in our study, the developmental history and clinical picture, including the "bizarre/psychotic" behaviour in some people, fitted best with autistic spectrum disorders. We do not argue that psychiatric conditions, such as schizophrenia, cannot occur in association with autistic disorders. The point of our paper is that catatonia can occur as a complication of autistic spectrum disorders alone.

Twenty-one individuals in our study had received psychotropic medication for possible psychiatric conditions, and two people were treated with electroconvulsive therapy, all without useful effect on the catatonic features. The side-effects of neuroleptic medication were considered as possible causes of the catatonia. Of the 21 individuals who were medicated 10 were given drugs only after the onset of catatonia. The temporal relationships were 
difficult to establish for the other 11 , but there was no clear evidence for cause and effect.

When considering aetiology, an important point is that many of the features described in discussions of catatonic phenomena are also characteristic of autistic disorders. This has interesting implications for the nature of autism and catatonia, and their relationship to each other and to other psychiatric conditions associated with impairments of motor function. This will be the subject of a separate paper.

L.Wing Centre for Social and Communication Disorders, Elliot House, II3 Masons Hill, Bromley, Kent BR2 9HT

A. Shah Leading Edge Psychology, I The Close, Dale Road, Purley, Surrey CR8 2EA

\section{In-patient detoxification after GHB dependence}

Gamma-hydroxybutyrate (GHB) occurs naturally in the human brain (Roth $\&$ Giarman, 1970). It has a structure similar to $\gamma$-aminobutyric acid and has been used as an anaesthetic and in the treatment of narcolepsy (Mamelak et al, 1986) and alcohol dependence (Gallimberti et al, 1989). There have been cases of dependence with withdrawal symptoms (Galloway et al, 1997). I believe this to be the first reported case of in-patient detoxification.

Between October and December 1999 two patients dependent on GHB were admitted to our in-patient unit for detoxification. This report summarises the case history of the first patient admitted. The second patient had a similar history.

A 43-year-old male reported using GHB for 2.5 years. The effects of use were reported as feelings of relaxation, inner well-being, increased appetite and short, restful periods of sleep. Starting doses of GHB were difficult to quantify as it is generally taken in liquid form. The patient said his dose was originally $15 \mathrm{ml}$ irregularly, but had increased to $30 \mathrm{ml}$ every 3 hours by the beginning of 1999. The patient reported withdrawal experiences such as feelings of panic, terror and anxiety, often with a tremor. Other autonomic features included diarrhoea. Adverse effects of GHB were reported, including accidental injury when intoxicated and episodes of bizarre behaviour. In-patient detoxification was thought necessary as both patients were unable to stop using GHB at home.
On admission to the unit physical examination was unremarkable apart from some features of anxiety. Physical investigations were normal. The patient was commenced on a diazepam reducing regime from $20 \mathrm{mg}$ over 11 days. Detoxification was uneventful. The patient was engaged in relapse prevention and referred to psychotherapy services.

Gamma-hydroxybutyrate has adverse effects but also produces euphoria and relaxation; GHB has abuse and dependence potential and so educational campaigns for the public may be important.

Gallimberti, L., Canton, G., Gentile, N., et al (1989) Gamma-hydroxybutyric acid for treatment of alcohol withdrawal syndrome. Lancet, ii, 787-789.

Galloway, G. P., Frederick, S. L., Staggers, F. E. Jr, et al (1997) Gamma-hydroxybutyrate: an emerging drug of abuse that causes physical dependence. Addiction, 92 . 89-96.

Mamelak, M., Scharf, M. \& Woods, M. (1986)

Treatment of narcolepsy with GHB. Sleep, 9. 285-289.

Roth, R. \& Giarman, N. (1970) Natural occurrence of GHB in mammalian brain. Biochemical Pharmacology, 19, 1087-1092.

G. Price South Sefton Community DrugsTeam, 18 Great Georges Road, Liverpool L22 IRB

\section{Suicide in psychiatric hospital in-patients in Ireland}

We were interested in the paper by Powell et al (2000) concerning suicide in psychiatric hospital in-patients. We conducted a 10-year review of the same phenomenon in Irish psychiatric hospitals (Corcoran \& Walsh, 1999). Although this was not a case-control study in the sense of Powell et al, the findings were very similar, particularly in respect of the high proportion of suicides $(70 \%)$ among in-patients who died 'off the premises' although 'on the books'. The Irish suicide rate among acute in-patients (i.e. those in hospital for less than one year), at 319 per 100000 of population $(v .118$ per 100000 for long-stay patients), is higher than that reported by Powell et al (137 per 100000 admissions).

However, the validity of calculating inpatient suicide rates on the basis of an admissions denominator is questionable. It ignores the length of time during which each individual is at risk during a hospital year, and is based on admissions rather than on the individuals contributing to these admissions. Many patients may have had several admissions in any given year. We approached the problem by the person-year method which takes into account not only the number of admissions, but also their mean length of stay and other data which were available to us from the National Psychiatric In-Patient Reporting System. Our paper discusses the imperfections of this approach.

Corcoran, E. \& Walsh, D. (1999) Suicide in psychiatric in-patients in Ireland. Irish Journal of Psychological Medicine, 16, |27-13|.

Powell, J., Geddes, J., Deeks, J., et al (2000) Suicide in psychiatric hospital in-patients. Risk factors and their predictive power. British Journal of Psychiatry, 176, 266-272.

E. Corcoran St Conal's Hospital, Letterkenny, Co. Donegal, Ireland

D.Walsh Inspector of Mental Hospitals and Health Research Board, 73 Lower Baggot Street, Dublin 2, Ireland

\section{Low blood pressure and depression in the elderly}

Paterniti et al (2000) report welcome prospective data showing that low blood pressure precedes depression in older people rather than vice versa. However, important alternative explanations for this effect require consideration before conclusions can be drawn regarding causality.

Poor physical health and disablement have been shown to be strong risk factors for incident depression in older people (Prince et al, 1997). A large prospective community study showed that low blood pressure was associated with raised mortality but that this effect was eliminated after adjustment for comorbid physical illness (Boshuizen et al, 1998). The same may be true for depression. Paterniti et al comment that the number of chronic diseases was measured in participants and was not associated with depressive symptoms or low blood pressure. However, it is the severity of individual conditions and resulting functional limitation which are likely to be most important in depression rather than the number of different conditions. Important confounding effects may therefore have been missed. Both depression and low blood pressure may also be secondary to early cognitive decline, although this is less likely to explain results in the age range of participants for this study.

In order to develop effective strategies for the prevention of depression in later life, 